Commun. Fac. Sci. Univ. Ank. Ser. A1 Math. Stat.

Volume 69, Number 1, Pages 49-72(2020)

DOI: $10.31801 /$ cfsuasmas. 542665

ISSN 1303-5991 E-ISSN 2618-6470

http://communications.science.ankara.edu.tr/index.php?series=A1

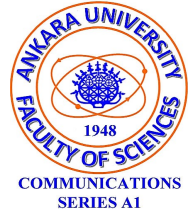

\title{
FURTHER INEQUALITIES FOR THE GENERALIZED $k$-g-FRACTIONAL INTEGRALS OF FUNCTIONS WITH BOUNDED VARIATION
}

\author{
SILVESTRU SEVER DRAGOMIR
}

ABSTRACT. Let $g$ be a strictly increasing function on $(a, b)$, having a continuous derivative $g^{\prime}$ on $(a, b)$. For the Lebesgue integrable function $f:(a, b) \rightarrow \mathbb{C}$, we define the $k$-g-left-sided fractional integral of $f$ by

$$
S_{k, g, a+} f(x)=\int_{a}^{x} k(g(x)-g(t)) g^{\prime}(t) f(t) d t, x \in(a, b]
$$

and the $k$-g-right-sided fractional integral of $f$ by

$$
S_{k, g, b-} f(x)=\int_{x}^{b} k(g(t)-g(x)) g^{\prime}(t) f(t) d t, x \in[a, b),
$$

where the kernel $k$ is defined either on $(0, \infty)$ or on $[0, \infty)$ with complex values and integrable on any finite subinterval.

In this paper we establish some new inequalities for the $k$ - $g$-fractional integrals of functions of bounded variation.Examples for the generalized left- and right-sided Riemann-Liouville fractional integrals of a function $f$ with respect to another function $g$ and a general exponential fractional integral are also provided.

\section{INTRODUCTION}

Assume that the kernel $k$ is defined either on $(0, \infty)$ or on $[0, \infty)$ with complex values and integrable on any finite subinterval. We define the function $K:[0, \infty) \rightarrow$ $\mathbb{C}$ by

$$
K(t):=\left\{\begin{array}{l}
\int_{0}^{t} k(s) d s \text { if } 0<t \\
0 \text { if } t=0 .
\end{array}\right.
$$

Received by the editors: March 21, 2019; Accepted: July 19, 2019.

2010 Mathematics Subject Classification. Primary 26D15, 26D10; Secondary 26D07, 26A33.

Key words and phrases. Generalized Riemann-Liouville fractional integrals, Hadamard fractional integrals, Functions of bounded variation, Ostrowski type inequalities, Trapezoid inequalities. 
SILVESTRU SEVER DRAGOMIR

As a simple example, if $k(t)=t^{\alpha-1}$ then for $\alpha \in(0,1)$ the function $k$ is defined on $(0, \infty)$ and $K(t):=\frac{1}{\alpha} t^{\alpha}$ for $t \in[0, \infty)$. If $\alpha \geq 1$, then $k$ is defined on $[0, \infty)$ and $K(t):=\frac{1}{\alpha} t^{\alpha}$ for $t \in[0, \infty)$.

Let $g$ be a strictly increasing function on $(a, b)$, having a continuous derivative $g^{\prime}$ on $(a, b)$. For the Lebesgue integrable function $f:(a, b) \rightarrow \mathbb{C}$, we define the $k$-g-left-sided fractional integral of $f$ by

$$
S_{k, g, a+} f(x)=\int_{a}^{x} k(g(x)-g(t)) g^{\prime}(t) f(t) d t, x \in(a, b]
$$

and the $k$-g-right-sided fractional integral of $f$ by

$$
S_{k, g, b-} f(x)=\int_{x}^{b} k(g(t)-g(x)) g^{\prime}(t) f(t) d t, x \in[a, b) .
$$

If we take $k(t)=\frac{1}{\Gamma(\alpha)} t^{\alpha-1}$, where $\Gamma$ is the Gamma function, then

$$
\begin{aligned}
S_{k, g, a+} f(x) & =\frac{1}{\Gamma(\alpha)} \int_{a}^{x}[g(x)-g(t)]^{\alpha-1} g^{\prime}(t) f(t) d t \\
& =: I_{a+, g}^{\alpha} f(x), a<x \leq b
\end{aligned}
$$

and

$$
\begin{aligned}
S_{k, g, b-} f(x) & =\frac{1}{\Gamma(\alpha)} \int_{x}^{b}[g(t)-g(x)]^{\alpha-1} g^{\prime}(t) f(t) d t \\
& =: I_{b-, g}^{\alpha} f(x), a \leq x<b,
\end{aligned}
$$

which are the generalized left- and right-sided Riemann-Liouville fractional integrals of a function $f$ with respect to another function $g$ on $[a, b]$ as defined in [23, p. 100].

For $g(t)=t$ in (4) we have the classical Riemann-Liouville fractional integrals while for the logarithmic function $g(t)=\ln t$ we have the Hadamard fractional integrals [23, p. 111]

$$
H_{a+}^{\alpha} f(x):=\frac{1}{\Gamma(\alpha)} \int_{a}^{x}\left[\ln \left(\frac{x}{t}\right)\right]^{\alpha-1} \frac{f(t) d t}{t}, 0 \leq a<x \leq b
$$

and

$$
H_{b-}^{\alpha} f(x):=\frac{1}{\Gamma(\alpha)} \int_{x}^{b}\left[\ln \left(\frac{t}{x}\right)\right]^{\alpha-1} \frac{f(t) d t}{t}, 0 \leq a<x<b .
$$

One can consider the function $g(t)=-t^{-1}$ and define the "Harmonic fractional integrals" by

$$
R_{a+}^{\alpha} f(x):=\frac{x^{1-\alpha}}{\Gamma(\alpha)} \int_{a}^{x} \frac{f(t) d t}{(x-t)^{1-\alpha} t^{\alpha+1}}, 0 \leq a<x \leq b
$$

and

$$
R_{b-}^{\alpha} f(x):=\frac{x^{1-\alpha}}{\Gamma(\alpha)} \int_{x}^{b} \frac{f(t) d t}{(t-x)^{1-\alpha} t^{\alpha+1}}, 0 \leq a<x<b .
$$


Also, for $g(t)=\exp (\beta t), \beta>0$, we can consider the " $\beta$-Exponential fractional integrals"

$$
E_{a+, \beta}^{\alpha} f(x):=\frac{\beta}{\Gamma(\alpha)} \int_{a}^{x}[\exp (\beta x)-\exp (\beta t)]^{\alpha-1} \exp (\beta t) f(t) d t,
$$

for $a<x \leq b$ and

$$
E_{b-, \beta}^{\alpha} f(x):=\frac{\beta}{\Gamma(\alpha)} \int_{x}^{b}[\exp (\beta t)-\exp (\beta x)]^{\alpha-1} \exp (\beta t) f(t) d t,
$$

for $a \leq x<b$.

If we take $g(t)=t$ in (1) and (2), then we can consider the following $k$-fractional integrals

$$
S_{k, a+} f(x)=\int_{a}^{x} k(x-t) f(t) d t, x \in(a, b]
$$

and

$$
S_{k, b-} f(x)=\int_{x}^{b} k(t-x) f(t) d t, x \in[a, b) .
$$

In 26, Raina studied a class of functions defined formally by

$$
\mathcal{F}_{\rho, \lambda}^{\sigma}(x):=\sum_{k=0}^{\infty} \frac{\sigma(k)}{\Gamma(\rho k+\lambda)} x^{k},|x|<R, \text { with } R>0
$$

for $\rho, \lambda>0$ where the coefficients $\sigma(k)$ generate a bounded sequence of positive real numbers. With the help of (13), Raina defined the following left-sided fractional integral operator

$$
\mathcal{J}_{\rho, \lambda, a+; w}^{\sigma} f(x):=\int_{a}^{x}(x-t)^{\lambda-1} \mathcal{F}_{\rho, \lambda}^{\sigma}\left(w(x-t)^{\rho}\right) f(t) d t, x>a
$$

where $\rho, \lambda>0, w \in \mathbb{R}$ and $f$ is such that the integral on the right side exists.

In [1, the right-sided fractional operator was also introduced as

$$
\mathcal{J}_{\rho, \lambda, b-; w}^{\sigma} f(x):=\int_{x}^{b}(t-x)^{\lambda-1} \mathcal{F}_{\rho, \lambda}^{\sigma}\left(w(t-x)^{\rho}\right) f(t) d t, x<b
$$

where $\rho, \lambda>0, w \in \mathbb{R}$ and $f$ is such that the integral on the right side exists. Several Ostrowski type inequalities were also established.

We observe that for $k(t)=t^{\lambda-1} \mathcal{F}_{\rho, \lambda}^{\sigma}\left(w t^{\rho}\right)$ we re-obtain the definitions of 14 ) and 15 from $(11)$ and 12 .

In [24, Kirane and Torebek introduced the following exponential fractional integrals

$$
\mathcal{T}_{a+}^{\alpha} f(x):=\frac{1}{\alpha} \int_{a}^{x} \exp \left\{-\frac{1-\alpha}{\alpha}(x-t)\right\} f(t) d t, x>a
$$

and

$$
\mathcal{T}_{b-}^{\alpha} f(x):=\frac{1}{\alpha} \int_{x}^{b} \exp \left\{-\frac{1-\alpha}{\alpha}(t-x)\right\} f(t) d t, x<b
$$


where $\alpha \in(0,1)$.

We observe that for $k(t)=\frac{1}{\alpha} \exp \left(-\frac{1-\alpha}{\alpha} t\right), t \in \mathbb{R}$ we re-obtain the definitions of (16) and (17) from (11) and (12).

Let $g$ be a strictly increasing function on $(a, b)$, having a continuous derivative $g^{\prime}$ on $(a, b)$. We can define the more general exponential fractional integrals

$$
\mathcal{T}_{g, a+}^{\alpha} f(x):=\frac{1}{\alpha} \int_{a}^{x} \exp \left\{-\frac{1-\alpha}{\alpha}(g(x)-g(t))\right\} g^{\prime}(t) f(t) d t, x>a
$$

and

$$
\mathcal{T}_{g, b-}^{\alpha} f(x):=\frac{1}{\alpha} \int_{x}^{b} \exp \left\{-\frac{1-\alpha}{\alpha}(g(t)-g(x))\right\} g^{\prime}(t) f(t) d t, x<b
$$

where $\alpha \in(0,1)$.

Let $g$ be a strictly increasing function on $(a, b)$, having a continuous derivative $g^{\prime}$ on $(a, b)$. Assume that $\alpha>0$. We can also define the logarithmic fractional integrals

$$
\mathcal{L}_{g, a+}^{\alpha} f(x):=\int_{a}^{x}(g(x)-g(t))^{\alpha-1} \ln (g(x)-g(t)) g^{\prime}(t) f(t) d t,
$$

for $0<a<x \leq b$ and

$$
\mathcal{L}_{g, b-}^{\alpha} f(x):=\int_{x}^{b}(g(t)-g(x))^{\alpha-1} \ln (g(t)-g(x)) g^{\prime}(t) f(t) d t,
$$

for $0<a \leq x<b$, where $\alpha>0$. These are obtained from (11) and 12 for the kernel $k(t)=t^{\alpha-1} \ln t, t>0$.

For $\alpha=1$ we get

$$
\mathcal{L}_{g, a+} f(x):=\int_{a}^{x} \ln (g(x)-g(t)) g^{\prime}(t) f(t) d t, 0<a<x \leq b
$$

and

$$
\mathcal{L}_{g, b-} f(x):=\int_{x}^{b} \ln (g(t)-g(x)) g^{\prime}(t) f(t) d t, 0<a \leq x<b .
$$

For $g(t)=t$, we have the simple forms

$$
\begin{gathered}
\mathcal{L}_{a+}^{\alpha} f(x):=\int_{a}^{x}(x-t)^{\alpha-1} \ln (x-t) f(t) d t, 0<a<x \leq b, \\
\mathcal{L}_{b-}^{\alpha} f(x):=\int_{x}^{b}(t-x)^{\alpha-1} \ln (t-x) f(t) d t, 0<a \leq x<b, \\
\mathcal{L}_{a+} f(x):=\int_{a}^{x} \ln (x-t) f(t) d t, 0<a<x \leq b
\end{gathered}
$$

and

$$
\mathcal{L}_{b-} f(x):=\int_{x}^{b} \ln (t-x) f(t) d t, 0<a \leq x<b .
$$

For several Ostrowski type inequalities for Riemann-Liouville fractional integrals see [2]-[17, 21]-34] and the references therein. 
For $k$ and $g$ as at the beginning of Introduction, we consider the mixed operator

$$
\begin{aligned}
& S_{k, g, a+, b-} f(x) \\
& :=\frac{1}{2}\left[S_{k, g, a+} f(x)+S_{k, g, b-} f(x)\right] \\
& =\frac{1}{2}\left[\int_{a}^{x} k(g(x)-g(t)) g^{\prime}(t) f(t) d t+\int_{x}^{b} k(g(t)-g(x)) g^{\prime}(t) f(t) d t\right]
\end{aligned}
$$

for the Lebesgue integrable function $f:(a, b) \rightarrow \mathbb{C}$ and $x \in(a, b)$.

We also define the function $\mathbf{K}:[0, \infty) \rightarrow[0, \infty)$ by

$$
\mathbf{K}(t):=\left\{\begin{array}{l}
\int_{0}^{t}|k(s)| d s \text { if } 0<t, \\
0 \text { if } t=0 .
\end{array}\right.
$$

In the recent paper 19] we obtained the following result for functions of bounded variation:

Theorem 1. Assume that the kernel $k$ is defined either on $(0, \infty)$ or on $[0, \infty)$ with complex values and integrable on any finite subinterval. Let $f:[a, b] \rightarrow \mathbb{C}$ be a function of bounded variation on $[a, b]$ and $g$ be a strictly increasing function on $(a, b)$, having a continuous derivative $g^{\prime}$ on $(a, b)$. Then we have the Ostrowski type inequality

$$
\begin{gathered}
\left|S_{k, g, a+, b-} f(x)-\frac{1}{2}[K(g(b)-g(x))+K(g(x)-g(a))] f(x)\right| \\
\leq \frac{1}{2}\left[\int_{x}^{b}|k(g(t)-g(x))| \bigvee_{x}^{t}(f) g^{\prime}(t) d t+\int_{a}^{x}|k(g(x)-g(t))| \bigvee_{t}^{x}(f) g^{\prime}(t) d t\right] \\
\leq \frac{1}{2}\left[\mathbf{K}(g(b)-g(x)) \bigvee_{x}^{b}(f)+\mathbf{K}(g(x)-g(a)) \bigvee_{a}^{x}(f)\right] \\
\leq \frac{1}{2}\left\{\begin{array}{l}
\max \{\mathbf{K}(g(b)-g(x)), \mathbf{K}(g(x)-g(a))\} \bigvee_{a}^{b}(f) ; \\
{\left[\mathbf{K}^{p}(g(b)-g(x))+\mathbf{K}^{p}(g(x)-g(a))\right]^{1 / p}\left(\left(\bigvee_{a}^{x}(f)\right)^{q}+\left(\bigvee_{x}^{b}(f)\right)^{q}\right)^{1 / q}} \\
\text { with } p, q>1, \frac{1}{p}+\frac{1}{q}=1 ; \\
{[\mathbf{K}(g(b)-g(x))+\mathbf{K}(g(x)-g(a))]\left[\frac{1}{2} \bigvee_{a}^{b}(f)+\frac{1}{2}\left|\bigvee_{a}^{x}(f)-\bigvee_{x}^{b}(f)\right|\right]}
\end{array}\right.
\end{gathered}
$$

and the trapezoid type inequality

$$
\left|S_{k, g, a+, b-} f(x)-\frac{1}{2}[K(g(b)-g(x)) f(b)+K(g(x)-g(a)) f(a)]\right|
$$




$$
\begin{aligned}
\leq \frac{1}{2}\left[\int_{a}^{x}|k(g(x)-g(t))| \bigvee_{a}^{t}(f) g^{\prime}(t) d t+\int_{x}^{b}|k(g(t)-g(x))| \bigvee_{t}^{b}(f) g^{\prime}(t) d t\right] \\
\leq \frac{1}{2}\left[\mathbf{K}(g(b)-g(x)) \bigvee_{x}^{b}(f)+\mathbf{K}(g(x)-g(a)) \bigvee_{a}^{x}(f)\right] \\
\leq \frac{1}{2}\left\{\begin{array}{l}
\max \{\mathbf{K}(g(b)-g(x)), \mathbf{K}(g(x)-g(a))\} \bigvee_{a}^{b}(f) ; \\
{\left[\mathbf{K}^{p}(g(b)-g(x))+\mathbf{K}^{p}(g(x)-g(a))\right]^{1 / p}} \\
\times\left(\left(\bigvee_{a}^{x}(f)\right)^{q}+\left(\bigvee_{x}^{b}(f)\right)^{q}\right)^{1 / q} \\
w i t h p, q>1, \frac{1}{p}+\frac{1}{q}=1 ; \\
{[\mathbf{K}(g(b)-g(x))+\mathbf{K}(g(x)-g(a))]} \\
\times\left[\frac{1}{2} \bigvee_{a}^{b}(f)+\frac{1}{2}\left|\bigvee_{a}^{x}(f)-\bigvee_{x}^{b}(f)\right|\right]
\end{array}\right.
\end{aligned}
$$

for any $x \in(a, b)$, where $\bigvee_{c}^{d}(f)$ denoted the total variation on the interval $[c, d]$.

Observe that

$$
S_{k, g, x+} f(b)=\int_{x}^{b} k(g(b)-g(t)) g^{\prime}(t) f(t) d t, x \in[a, b)
$$

and

$$
S_{k, g, x-} f(a)=\int_{a}^{x} k(g(t)-g(a)) g^{\prime}(t) f(t) d t, x \in(a, b] .
$$

We can define also the mixed operator

$$
\begin{aligned}
& \breve{S}_{k, g, a+, b-} f(x) \\
& :=\frac{1}{2}\left[S_{k, g, x+} f(b)+S_{k, g, x-} f(a)\right] \\
& =\frac{1}{2}\left[\int_{x}^{b} k(g(b)-g(t)) g^{\prime}(t) f(t) d t+\int_{a}^{x} k(g(t)-g(a)) g^{\prime}(t) f(t) d t\right]
\end{aligned}
$$

for any $x \in(a, b)$.

In this paper we establish some inequalities for the $k$ - $g$-fractional integrals of functions with bounded variation $f:[a, b] \rightarrow \mathbb{C}$ that provide error bounds in approximating the composite operators $S_{k, g, a+, b-} f$ and $\breve{S}_{k, g, a+, b-} f$ in terms of the double trapezoid rule

$$
\frac{1}{2}\left[\frac{f(x)+f(b)}{2} K(g(b)-g(x))+\frac{f(a)+f(x)}{2} K(g(x)-g(a))\right], x \in(a, b) .
$$

Examples for the generalized left- and right-sided Riemann-Liouville fractional integrals of a function $f$ with respect to another function $g$ and a general exponential fractional integral are also provided. 


\section{Further Inequalities for Functions of BV}

The following two parameters representation for the operators $S_{k, g, a+, b-}$ and $\breve{S}_{k, g, a+, b-}$ hold [20]:

Lemma 2. Assume that the kernel $k$ is defined either on $(0, \infty)$ or on $[0, \infty)$ with complex values and integrable on any finite subinterval. Let $f:[a, b] \rightarrow \mathbb{C}$ be an integrable function on $[a, b]$ and $g$ be a strictly increasing function on $(a, b)$, having a continuous derivative $g^{\prime}$ on $(a, b)$. Then

$$
\begin{aligned}
S_{k, g, a+, b-} f(x) & =\frac{1}{2}[\gamma K(g(b)-g(x))+\lambda K(g(x)-g(a))] \\
& +\frac{1}{2} \int_{a}^{x} k(g(x)-g(t)) g^{\prime}(t)[f(t)-\lambda] d t \\
& +\frac{1}{2} \int_{x}^{b} k(g(t)-g(x)) g^{\prime}(t)[f(t)-\gamma] d t
\end{aligned}
$$

and

$$
\begin{aligned}
\breve{S}_{k, g, a+, b-} f(x) & =\frac{1}{2}[\lambda K(g(b)-g(x))+\gamma K(g(x)-g(a))] \\
& +\frac{1}{2} \int_{a}^{x} k(g(t)-g(a)) g^{\prime}(t)[f(t)-\gamma] d t \\
& +\frac{1}{2} \int_{x}^{b} k(g(b)-g(t)) g^{\prime}(t)[f(t)-\lambda] d t
\end{aligned}
$$

for $x \in(a, b)$ and for any $\lambda, \gamma \in \mathbb{C}$.

Proof. We have, by taking the derivative over $t$ and using the chain rule, that

$$
[K(g(x)-g(t))]^{\prime}=K^{\prime}(g(x)-g(t))(g(x)-g(t))^{\prime}=-k(g(x)-g(t)) g^{\prime}(t)
$$

for $t \in(a, x)$ and

$$
[K(g(t)-g(x))]^{\prime}=K^{\prime}(g(t)-g(x))(g(t)-g(x))^{\prime}=k(g(t)-g(x)) g^{\prime}(t)
$$

for $t \in(x, b)$.

Therefore, for any $\lambda, \gamma \in \mathbb{C}$ we have

$$
\begin{aligned}
& \int_{a}^{x} k(g(x)-g(t)) g^{\prime}(t)[f(t)-\lambda] d t \\
& =\int_{a}^{x} k(g(x)-g(t)) g^{\prime}(t) f(t) d t-\lambda \int_{a}^{x} k(g(x)-g(t)) g^{\prime}(t) d t \\
& =S_{k, g, a+} f(x)+\lambda \int_{a}^{x}[K(g(x)-g(t))]^{\prime} d t \\
& =S_{k, g, a+} f(x)+\left.\lambda[K(g(x)-g(t))]\right|_{a} ^{x}=S_{k, g, a+} f(x)-\lambda K(g(x)-g(a))
\end{aligned}
$$


and

$$
\begin{aligned}
& \int_{x}^{b} k(g(t)-g(x)) g^{\prime}(t)[f(t)-\gamma] d t \\
& =\int_{x}^{b} k(g(t)-g(x)) g^{\prime}(t) f(t) d t-\gamma \int_{x}^{b} k(g(t)-g(x)) g^{\prime}(t) d t \\
& =S_{k, g, b-} f(x)-\gamma \int_{x}^{b}[K(g(t)-g(x))]^{\prime} d t \\
& =S_{k, g, b-} f(x)-\left.\gamma[K(g(t)-g(x))]\right|_{x} ^{b}=S_{k, g, b-} f(x)-\gamma K(g(b)-g(x))
\end{aligned}
$$

for $x \in(a, b)$.

If we add the equalities (36) and (37) and divide by 2 then we get the desired result (34).

Moreover, by taking the derivative over $t$ and using the chain rule, we have that

$$
[K(g(b)-g(t))]^{\prime}=K^{\prime}(g(b)-g(t))(g(b)-g(t))^{\prime}=-k(g(b)-g(t)) g^{\prime}(t)
$$

for $t \in(x, b)$ and

$$
[K(g(t)-g(a))]^{\prime}=K^{\prime}(g(t)-g(a))(g(t)-g(a))^{\prime}=k(g(t)-g(a)) g^{\prime}(t)
$$

for $t \in(a, x)$.

For any $\lambda, \gamma \in \mathbb{C}$ we have

$$
\begin{aligned}
& \int_{x}^{b} k(g(b)-g(t)) g^{\prime}(t)[f(t)-\lambda] d t \\
& =\int_{x}^{b} k(g(b)-g(t)) g^{\prime}(t) f(t) d t-\lambda \int_{x}^{b} k(g(b)-g(t)) g^{\prime}(t) d t \\
& =S_{k, g, x+} f(b)+\lambda \int_{x}^{b}[K(g(b)-g(t))]^{\prime} d t \\
& =S_{k, g, x+} f(b)-\lambda K(g(b)-g(x))
\end{aligned}
$$

and

$$
\begin{aligned}
& \int_{a}^{x} k(g(t)-g(a)) g^{\prime}(t)[f(t)-\gamma] d t \\
& =\int_{a}^{x} k(g(t)-g(a)) g^{\prime}(t) f(t) d t-\gamma \int_{a}^{x} k(g(t)-g(a)) g^{\prime}(t) d t \\
& =\int_{a}^{x} k(g(t)-g(a)) g^{\prime}(t) f(t) d t-\gamma \int_{a}^{x}[K(g(t)-g(a))]^{\prime} d t \\
& =\int_{a}^{x} k(g(t)-g(a)) g^{\prime}(t) f(t) d t-\gamma K(g(x)-g(a))
\end{aligned}
$$

for $x \in(a, b)$.

If we add the equalities (38) and $(39)$ and divide by 2 then we get the desired result (35). 
If $g$ is a function which maps an interval $I$ of the real line to the real numbers, and is both continuous and injective then we can define the $g$-mean of two numbers $a, b \in I$ as

$$
M_{g}(a, b):=g^{-1}\left(\frac{g(a)+g(b)}{2}\right) .
$$

If $I=\mathbb{R}$ and $g(t)=t$ is the identity function, then $M_{g}(a, b)=A(a, b):=\frac{a+b}{2}$, the arithmetic mean. If $I=(0, \infty)$ and $g(t)=\ln t$, then $M_{g}(a, b)=G(a, b):=\sqrt{a b}$, the geometric mean. If $I=(0, \infty)$ and $g(t)=\frac{1}{t}$, then $M_{g}(a, b)=H(a, b):=$ $\frac{2 a b}{a+b}$, the harmonic mean. If $I=(0, \infty)$ and $g(t)=t^{p}, p \neq 0$, then $M_{g}(a, b)=$ $M_{p}(a, b):=\left(\frac{a^{p}+b^{p}}{2}\right)^{1 / p}$, the power mean with exponent $p$. Finally, if $I=\mathbb{R}$ and $g(t)=\exp t$, then

$$
M_{g}(a, b)=L M E(a, b):=\ln \left(\frac{\exp a+\exp b}{2}\right),
$$

the LogMeanExp function.

Using the $g$-mean of two numbers we can introduce

$$
\begin{aligned}
P_{k, g, a+, b-} f & :=S_{k, g, a+, b-} f\left(M_{g}(a, b)\right) \\
& =\frac{1}{2} \int_{a}^{M_{g}(a, b)} k\left(\frac{g(a)+g(b)}{2}-g(t)\right) g^{\prime}(t) f(t) d t \\
& +\frac{1}{2} \int_{M_{g}(a, b)}^{b} k\left(g(t)-\frac{g(a)+g(b)}{2}\right) g^{\prime}(t) f(t) d t .
\end{aligned}
$$

Using the representation (34) we have

$$
\begin{aligned}
P_{k, g, a+, b-} f & =K\left(\frac{g(b)-g(a)}{2}\right) \frac{\gamma+\lambda}{2} \\
& +\frac{1}{2} \int_{a}^{M_{g}(a, b)} k\left(\frac{g(a)+g(b)}{2}-g(t)\right) g^{\prime}(t)[f(t)-\lambda] d t \\
& +\frac{1}{2} \int_{M_{g}(a, b)}^{b} k\left(g(t)-\frac{g(a)+g(b)}{2}\right) g^{\prime}(t)[f(t)-\gamma] d t
\end{aligned}
$$

for any $\lambda, \gamma \in \mathbb{C}$.

Also, if

$$
\begin{aligned}
\breve{P}_{k, g, a+, b-} f & :=\breve{S}_{k, g, a+, b-} f\left(M_{g}(a, b)\right) \\
& =\frac{1}{2} \int_{M_{g}(a, b)}^{b} k(g(b)-g(t)) g^{\prime}(t) f(t) d t \\
& +\frac{1}{2} \int_{a}^{M_{g}(a, b)} k(g(t)-g(a)) g^{\prime}(t) f(t) d t .
\end{aligned}
$$


then by 35 we get

$$
\begin{aligned}
\breve{P}_{k, g, a+, b-} f & =K\left(\frac{g(b)-g(a)}{2}\right) \frac{\gamma+\lambda}{2} \\
& +\frac{1}{2} \int_{a}^{M_{g}(a, b)} k(g(t)-g(a)) g^{\prime}(t)[f(t)-\gamma] d t \\
& +\frac{1}{2} \int_{M_{g}(a, b)}^{b} k(g(b)-g(t)) g^{\prime}(t)[f(t)-\lambda] d t
\end{aligned}
$$

for any $\lambda, \gamma \in \mathbb{C}$.

Theorem 3. Assume that the kernel $k$ is defined either on $(0, \infty)$ or on $[0, \infty)$ with complex values and integrable on any finite subinterval. Let $f:[a, b] \rightarrow \mathbb{C}$ be a function of bounded variation on $[a, b]$ and $g$ be a strictly increasing function on $(a, b)$, having a continuous derivative $g^{\prime}$ on $(a, b)$. Then we have the double trapezoid inequalities

$$
\begin{aligned}
& \mid S_{k, g, a+, b-} f(x) \\
& -\frac{1}{2}\left[\frac{f(x)+f(b)}{2} K(g(b)-g(x))+\frac{f(a)+f(x)}{2} K(g(x)-g(a))\right] \mid \\
& \leq \frac{1}{4}\left[\mathbf{K}(g(x)-g(a)) \bigvee_{a}^{x}(f)+\mathbf{K}(g(b)-g(x)) \bigvee_{x}^{b}(f)\right] \\
& \leq \frac{1}{4}\left\{\begin{array}{l}
\max \{\mathbf{K}(g(b)-g(x)), \mathbf{K}(g(x)-g(a))\} \bigvee_{a}^{b}(f) ; \\
{\left[\mathbf{K}^{p}(g(b)-g(x))+\mathbf{K}^{p}(g(x)-g(a))\right]^{1 / p}\left(\left(\bigvee_{a}^{x}(f)\right)^{q}+\left(\bigvee_{x}^{b}(f)\right)^{q}\right)^{1 / q}} \\
\text { with } p, q>1, \frac{1}{p}+\frac{1}{q}=1 ; \\
{[\mathbf{K}(g(b)-g(x))+\mathbf{K}(g(x)-g(a))]\left[\frac{1}{2} \bigvee_{a}^{b}(f)+\frac{1}{2}\left|\bigvee_{a}^{x}(f)-\bigvee_{x}^{b}(f)\right|\right]}
\end{array}\right.
\end{aligned}
$$

and

$$
\begin{aligned}
& \mid \breve{S}_{k, g, a+, b-} f(x) \\
& -\frac{1}{2}\left[\frac{f(x)+f(b)}{2} K(g(b)-g(x))+\frac{f(a)+f(x)}{2} K(g(x)-g(a))\right] \mid \\
& \leq \frac{1}{4}\left[\mathbf{K}(g(x)-g(a)) \bigvee_{a}^{x}(f)+\mathbf{K}(g(b)-g(x)) \bigvee_{x}^{b}(f)\right]
\end{aligned}
$$




$$
\leq \frac{1}{4}\left\{\begin{array}{l}
\max \{\mathbf{K}(g(b)-g(x)), \mathbf{K}(g(x)-g(a))\} \bigvee_{a}^{b}(f) ; \\
{\left[\mathbf{K}^{p}(g(b)-g(x))+\mathbf{K}^{p}(g(x)-g(a))\right]^{1 / p}\left(\left(\bigvee_{a}^{x}(f)\right)^{q}+\left(\bigvee_{x}^{b}(f)\right)^{q}\right)^{1 / q}} \\
\text { with } p, q>1, \frac{1}{p}+\frac{1}{q}=1 ; \\
{[\mathbf{K}(g(b)-g(x))+\mathbf{K}(g(x)-g(a))]\left[\frac{1}{2} \bigvee_{a}^{b}(f)+\frac{1}{2}\left|\bigvee_{a}^{x}(f)-\bigvee_{x}^{b}(f)\right|\right]}
\end{array}\right.
$$

for $x \in(a, b)$.

Proof. Using the identity 34 for $\lambda=\frac{f(a)+f(x)}{2}$ and $\gamma=\frac{f(x)+f(b)}{2}$ we have

$$
\begin{aligned}
& S_{k, g, a+, b-} f(x) \\
& =\frac{1}{2}\left[\frac{f(x)+f(b)}{2} K(g(b)-g(x))+\frac{f(a)+f(x)}{2} K(g(x)-g(a))\right] \\
& +\frac{1}{2} \int_{a}^{x} k(g(x)-g(t)) g^{\prime}(t)\left[f(t)-\frac{f(a)+f(x)}{2}\right] d t \\
& +\frac{1}{2} \int_{x}^{b} k(g(t)-g(x)) g^{\prime}(t)\left[f(t)-\frac{f(x)+f(b)}{2}\right] d t
\end{aligned}
$$

for $x \in(a, b)$.

Since $f$ is of bounded variation, then

$$
\begin{aligned}
\left|f(t)-\frac{f(a)+f(x)}{2}\right| & =\left|\frac{f(t)-f(a)+f(t)-f(x)}{2}\right| \\
& \leq \frac{1}{2}[|f(t)-f(a)|+|f(x)-f(t)|] \leq \frac{1}{2} \bigvee_{a}^{x}(f)
\end{aligned}
$$

and

$$
\begin{aligned}
\left|f(t)-\frac{f(x)+f(b)}{2}\right| & =\left|\frac{f(t)-f(x)+f(t)-f(b)}{2}\right| \\
& \leq \frac{1}{2}[|f(t)-f(x)|+|f(b)-f(t)|] \leq \frac{1}{2} \bigvee_{x}^{b}(f)
\end{aligned}
$$

for $x \in(a, b)$.

Using the equality (46) we have

$$
\begin{aligned}
& \mid S_{k, g, a+, b-} f(x) \\
& -\frac{1}{2}\left[\frac{f(x)+f(b)}{2} K(g(b)-g(x))+\frac{f(a)+f(x)}{2} K(g(x)-g(a))\right] \mid \\
& \quad \leq \frac{1}{2}\left|\int_{a}^{x} k(g(x)-g(t)) g^{\prime}(t)\left[f(t)-\frac{f(a)+f(x)}{2}\right] d t\right|
\end{aligned}
$$




$$
\begin{aligned}
+ & \frac{1}{2}\left|\int_{x}^{b} k(g(t)-g(x)) g^{\prime}(t)\left[f(t)-\frac{f(x)+f(b)}{2}\right] d t\right| \\
\leq & \frac{1}{2} \int_{a}^{x}|k(g(x)-g(t))|\left|f(t)-\frac{f(a)+f(x)}{2}\right| g^{\prime}(t) d t \\
& +\frac{1}{2} \int_{x}^{b}|k(g(t)-g(x))|\left|f(t)-\frac{f(x)+f(b)}{2}\right| g^{\prime}(t) d t \\
\leq \frac{1}{4}\left[\bigvee_{a}^{x}(f) \int_{a}^{x}|k(g(x)-g(t))| g^{\prime}(t) d t+\bigvee_{x}^{b}(f) \int_{x}^{b}|k(g(t)-g(x))| g^{\prime}(t) d t\right] & =: B(x) \quad(47)
\end{aligned}
$$

for $x \in(a, b)$.

We have, by taking the derivative over $t$ and using the chain rule, that

$$
[\mathbf{K}(g(x)-g(t))]^{\prime}=\mathbf{K}^{\prime}(g(x)-g(t))(g(x)-g(t))^{\prime}=-|k(g(x)-g(t))| g^{\prime}(t)
$$

for $t \in(a, x)$ and

$$
[\mathbf{K}(g(t)-g(x))]^{\prime}=\mathbf{K}^{\prime}(g(t)-g(x))(g(t)-g(x))^{\prime}=|k(g(t)-g(x))| g^{\prime}(t)
$$

for $t \in(x, b)$.

Then

$$
\int_{a}^{x}|k(g(x)-g(t))| g^{\prime}(t) d t=-\int_{a}^{x}[\mathbf{K}(g(x)-g(t))]^{\prime} d t=\mathbf{K}(g(x)-g(a))
$$

and

$$
\int_{x}^{b}|k(g(t)-g(x))| g^{\prime}(t) d t=\int_{x}^{b}[\mathbf{K}(g(t)-g(x))]^{\prime} d t=\mathbf{K}(g(b)-g(x)) .
$$

Therefore

$$
\begin{aligned}
B(x) & =\frac{1}{4}\left[\bigvee_{a}^{x}(f) \int_{a}^{x}|k(g(x)-g(t))| g^{\prime}(t) d t+\bigvee_{x}^{b}(f) \int_{x}^{b}|k(g(t)-g(x))| g^{\prime}(t) d t\right] \\
& =\frac{1}{4}\left[\mathbf{K}(g(x)-g(a)) \bigvee_{a}^{x}(f)+\mathbf{K}(g(b)-g(x)) \bigvee_{x}^{b}(f)\right] .
\end{aligned}
$$

The last part of (44) is obvious by making use of the elementary Hölder type inequalities for positive real numbers $c, d, m, n \geq 0$

$$
m c+n d \leq\left\{\begin{array}{l}
\max \{m, n\}(c+d) ; \\
\left(m^{p}+n^{p}\right)^{1 / p}\left(c^{q}+d^{q}\right)^{1 / q} \text { with } p, q>1, \frac{1}{p}+\frac{1}{q}=1 .
\end{array}\right.
$$

Using the identity 35 for $\lambda=\frac{f(x)+f(b)}{2}$ and $\gamma=\frac{f(x)+f(a)}{2}$ we also have $\mid \breve{S}_{k, g, a+, b-} f(x)$ 


$$
\begin{gathered}
-\frac{1}{2}\left[\frac{f(x)+f(b)}{2} K(g(b)-g(x))+\frac{f(x)+f(a)}{2} K(g(x)-g(a))\right] \mid \\
\leq \frac{1}{2} \int_{a}^{x}|k(g(t)-g(a))|\left|f(t)-\frac{f(x)+f(a)}{2}\right| g^{\prime}(t) d t \\
\quad+\frac{1}{2} \int_{x}^{b}|k(g(b)-g(t))|\left|f(t)-\frac{f(x)+f(b)}{2}\right| g^{\prime}(t) d t \\
\leq \frac{1}{4} \bigvee_{a}^{x}(f) \int_{a}^{x}|k(g(t)-g(a))| g^{\prime}(t) d t+\frac{1}{4} \bigvee_{x}^{b}(f) \int_{x}^{b}|k(g(b)-g(t))| g^{\prime}(t) d t \\
=: C(x) .
\end{gathered}
$$

We also have, by taking the derivative over $t$ and using the chain rule, that

$$
[\mathbf{K}(g(b)-g(t))]^{\prime}=\mathbf{K}^{\prime}(g(b)-g(t))(g(b)-g(t))^{\prime}=-|k(g(b)-g(t))| g^{\prime}(t)
$$

for $t \in(x, b)$ and

$$
[\mathbf{K}(g(t)-g(a))]^{\prime}=\mathbf{K}^{\prime}(g(t)-g(a))(g(t)-g(a))^{\prime}=|k(g(t)-g(a))| g^{\prime}(t)
$$

for $t \in(a, x)$.

Therefore

$$
\int_{a}^{x}|k(g(t)-g(a))| g^{\prime}(t) d t=\mathbf{K}(g(x)-g(a))
$$

and

$$
\int_{x}^{b}|k(g(b)-g(t))| g^{\prime}(t) d t=\mathbf{K}(g(b)-g(x))
$$

giving that

$$
C(x)=\frac{1}{4} \bigvee_{a}^{x}(f) \mathbf{K}(g(x)-g(a))+\frac{1}{4} \bigvee_{x}^{b}(f) \mathbf{K}(g(b)-g(x))
$$

for $x \in(a, b)$, and the inequality 45 is thus proved.

Corollary 4. With the assumptions of Theorem 3 we have

$$
\begin{aligned}
& \left|P_{k, g, a+, b-} f-\frac{1}{2} K\left(\frac{g(b)-g(a)}{2}\right)\left[f\left(M_{g}(a, b)\right)+\frac{f(a)+f(b)}{2}\right]\right| \\
& \leq \frac{1}{4} \mathbf{K}\left(\frac{g(b)-g(a)}{2}\right) \bigvee_{a}^{b}(f)
\end{aligned}
$$

and

$$
\begin{aligned}
& \left|\breve{P}_{k, g, a+, b-} f-\frac{1}{2} K\left(\frac{g(b)-g(a)}{2}\right)\left[f\left(M_{g}(a, b)\right)+\frac{f(a)+f(b)}{2}\right]\right| \\
& \leq \frac{1}{4} \mathbf{K}\left(\frac{g(b)-g(a)}{2}\right) \bigvee_{a}^{b}(f) .
\end{aligned}
$$


If we take $x=\frac{a+b}{2}$ in 44 and 45 , then we get

$$
\begin{aligned}
& \mid S_{k, g, a+, b-} f\left(\frac{a+b}{2}\right)-\frac{f\left(\frac{a+b}{2}\right)+f(b)}{4} K\left(g(b)-g\left(\frac{a+b}{2}\right)\right) \\
& -\frac{f(a)+f\left(\frac{a+b}{2}\right)}{4} K\left(g\left(\frac{a+b}{2}\right)-g(a)\right) \mid \\
& \leq \frac{1}{4}\left[\mathbf{K}\left(g\left(\frac{a+b}{2}\right)-g(a)\right) \bigvee_{a}^{\frac{a+b}{2}}(f)+\mathbf{K}\left(g(b)-g\left(\frac{a+b}{2}\right)\right) \bigvee_{\frac{a+b}{2}}^{b}(f)\right] \\
& \leq \frac{1}{4}\left\{\begin{array}{l}
\max \left\{\mathbf{K}\left(g(b)-g\left(\frac{a+b}{2}\right)\right), \mathbf{K}\left(g\left(\frac{a+b}{2}\right)-g(a)\right)\right\} \bigvee_{a}^{b}(f) ; \\
{\left[\mathbf{K}^{p}\left(g(b)-g\left(\frac{a+b}{2}\right)\right)+\mathbf{K}^{p}\left(g\left(\frac{a+b}{2}\right)-g(a)\right)\right]^{1 / p}} \\
\left(\left(\bigvee_{a}^{\frac{a+b}{2}}(f)\right)^{q}+\left(\bigvee_{\frac{a+b}{2}}^{b}(f)\right)^{q}\right)^{1 / q} \\
\operatorname{with} p, q>1, \frac{1}{p}+\frac{1}{q}=1 ; \\
{\left[\mathbf{K}\left(g(b)-g\left(\frac{a+b}{2}\right)\right)+\mathbf{K}\left(g\left(\frac{a+b}{2}\right)-g(a)\right)\right]} \\
{\left[\frac{1}{2} \bigvee_{a}^{b}(f)+\frac{1}{2}\left|\bigvee_{a}^{\frac{a+b}{2}}(f)-\bigvee_{\frac{a+b}{2}}^{b}(f)\right|\right]}
\end{array}\right.
\end{aligned}
$$

and

$$
\begin{gathered}
\mid \breve{S}_{k, g, a+, b-} f\left(\frac{a+b}{2}\right)-\frac{f\left(\frac{a+b}{2}\right)+f(b)}{4} K\left(g(b)-g\left(\frac{a+b}{2}\right)\right) \\
-\frac{f(a)+f\left(\frac{a+b}{2}\right)}{4} K\left(g\left(\frac{a+b}{2}\right)-g(a)\right) \mid \\
\leq \frac{1}{4}\left[\mathbf{K}\left(g\left(\frac{a+b}{2}\right)-g(a)\right) \bigvee_{a}^{\frac{a+b}{2}}(f)+\mathbf{K}\left(g(b)-g\left(\frac{a+b}{2}\right)\right) \bigvee_{x}^{b}(f)\right]
\end{gathered}
$$




$$
\leq \frac{1}{4}\left\{\begin{array}{l}
\max \left\{\mathbf{K}\left(g(b)-g\left(\frac{a+b}{2}\right)\right), \mathbf{K}\left(g\left(\frac{a+b}{2}\right)-g(a)\right)\right\} \bigvee_{a}^{b}(f) ; \\
{\left[\mathbf{K}^{p}\left(g(b)-g\left(\frac{a+b}{2}\right)\right)+\mathbf{K}^{p}\left(g\left(\frac{a+b}{2}\right)-g(a)\right)\right]^{1 / p}} \\
\left(\left(\bigvee_{a}^{\frac{a+b}{2}}(f)\right)^{q}+\left(\bigvee_{\frac{a+b}{2}}^{b}(f)\right)^{q}\right)^{1 / q} \\
\text { with } p, q>1, \frac{1}{p}+\frac{1}{q}=1 ; \\
{\left[\mathbf{K}\left(g(b)-g\left(\frac{a+b}{2}\right)\right)+\mathbf{K}\left(g\left(\frac{a+b}{2}\right)-g(a)\right)\right]} \\
{\left[\frac{1}{2} \bigvee_{a}^{b}(f)+\frac{1}{2}\left|\bigvee_{a}^{\frac{a+b}{2}}(f)-\bigvee_{\frac{a+b}{2}}^{b}(f)\right|\right]}
\end{array}\right.
$$

for $x \in(a, b)$.

We use the classical Lebesgue $p$-norms defined as

$$
\|h\|_{[c, d], \infty}:=\operatorname{essup}_{s \in[c, d]}|h(s)|
$$

and

$$
\|h\|_{[c, d], p}:=\left(\int_{c}^{d}|h(s)|^{p} d s\right)^{1 / p}, p \geq 1 .
$$

Using Hölder's integral inequality we have for $t>0$ that

$$
K(t)=\int_{0}^{t}|k(s)| d s \leq\left\{\begin{array}{l}
t\|k\|_{[0, t], \infty} \text { if } k \in L_{\infty}[0, t] \\
t^{1 / p}\|k\|_{[0, t], q} \text { if } k \in L_{q}[0, t], p, q>1, \frac{1}{p}+\frac{1}{q}=1 .
\end{array}\right.
$$

Therefore by the first inequality in 44 and 45 we get for $p, q>1, \frac{1}{p}+\frac{1}{q}=1$

$\mid S_{k, g, a+, b-} f(x)$

$$
\begin{aligned}
&-\frac{1}{2}\left[\frac{f(x)+f(b)}{2} K\right.\left.(g(b)-g(x))+\frac{f(a)+f(x)}{2} K(g(x)-g(a))\right] \mid \\
& \leq \frac{1}{4} \bigvee_{a}^{x}(f)\left\{\begin{array}{l}
(g(x)-g(a))\|k\|_{[0, g(x)-g(a)], \infty} \\
(g(x)-g(a))^{1 / p}\|k\|_{[0, g(x)-g(a)], q}
\end{array}\right. \\
&+\frac{1}{4} \bigvee_{x}^{b}(f)\left\{\begin{array}{l}
(g(b)-g(x))\|k\|_{[0, g(b)-g(x)], \infty} \\
(g(b)-g(x))^{1 / p}\|k\|_{[0, g(b)-g(x)], q}
\end{array}\right.
\end{aligned}
$$

and

$$
\begin{aligned}
& \mid \breve{S}_{k, g, a+, b-} f(x) \\
& \quad-\frac{1}{2}\left[\frac{f(x)+f(b)}{2} K(g(b)-g(x))+\frac{f(a)+f(x)}{2} K(g(x)-g(a))\right] \mid
\end{aligned}
$$




$$
\begin{aligned}
& \leq \frac{1}{4} \bigvee_{a}^{x}(f)\left\{\begin{array}{l}
(g(x)-g(a))\|k\|_{[0, g(x)-g(a)], \infty} \\
(g(x)-g(a))^{1 / p}\|k\|_{[0, g(x)-g(a)], q}
\end{array}\right. \\
&+\frac{1}{4} \bigvee_{x}^{b}(f)\left\{\begin{array}{l}
(g(b)-g(x))\|k\|_{[0, g(b)-g(x)], \infty} \\
(g(b)-g(x))^{1 / p}\|k\|_{[0, g(b)-g(x)], q}
\end{array}\right.
\end{aligned}
$$

for $x \in(a, b)$.

From 48 and 49 we also have for $p, q>1, \frac{1}{p}+\frac{1}{q}=1$ that

$$
\begin{aligned}
\left|P_{k, g, a+, b-} f-\frac{1}{2} K\left(\frac{g(b)-g(a)}{2}\right)\left[f\left(M_{g}(a, b)\right)+\frac{f(a)+f(b)}{2}\right]\right| \\
\leq \frac{1}{4} \bigvee_{a}^{b}(f)\left\{\begin{array}{l}
\left(\frac{g(b)-g(a)}{2}\right)\|k\|_{\left[0, \frac{g(b)-g(a)}{2}\right], \infty} \\
\left(\frac{g(b)-g(a)}{2}\right)^{1 / p}\|k\|_{\left[0, \frac{g(b)-g(a)}{2}\right], q}
\end{array}\right.
\end{aligned}
$$

and

$$
\begin{aligned}
& \mid \breve{P}_{k, g, a+, b-} f-\frac{1}{2} K\left(\frac{g(b)-g(a)}{2}\right) {\left[f\left(M_{g}(a, b)\right)+\frac{f(a)+f(b)}{2}\right] \mid } \\
& \leq \frac{1}{4} \bigvee_{a}^{b}(f)\left\{\begin{array}{l}
\left(\frac{g(b)-g(a)}{2}\right)\|k\|_{\left[0, \frac{g(b)-g(a)}{2}\right], \infty} \\
\left(\frac{g(b)-g(a)}{2}\right)^{1 / p}\|k\|_{\left[0, \frac{g(b)-g(a)}{2}\right], q}
\end{array}\right.
\end{aligned}
$$

3. Applications for Generalized Riemann-Liouville Fractional INTEGRALS

If we take $k(t)=\frac{1}{\Gamma(\alpha)} t^{\alpha-1}$, where $\Gamma$ is the Gamma function, then

$$
S_{k, g, a+} f(x)=I_{a+, g}^{\alpha} f(x):=\frac{1}{\Gamma(\alpha)} \int_{a}^{x}[g(x)-g(t)]^{\alpha-1} g^{\prime}(t) f(t) d t
$$

for $a<x \leq b$ and

$$
S_{k, g, b-} f(x)=I_{b-, g}^{\alpha} f(x):=\frac{1}{\Gamma(\alpha)} \int_{x}^{b}[g(t)-g(x)]^{\alpha-1} g^{\prime}(t) f(t) d t
$$

for $a \leq x<b$, which are the generalized left- and right-sided Riemann-Liouville fractional integrals of a function $f$ with respect to another function $g$ on $[a, b]$ as defined in [23, p. 100].

We consider the mixed operators

$$
I_{g, a+, b-}^{\alpha} f(x):=\frac{1}{2}\left[I_{a+, g}^{\alpha} f(x)+I_{b-, g}^{\alpha} f(x)\right]
$$


and

$$
\breve{I}_{g, a+, b-}^{\alpha} f(x):=\frac{1}{2}\left[I_{x+, g}^{\alpha} f(b)+I_{x-, g}^{\alpha} f(a)\right]
$$

for $x \in(a, b)$.

We observe that for $\alpha>0$ we have

$$
K(t)=\frac{1}{\Gamma(\alpha)} \int_{0}^{t} s^{\alpha-1} d s=\frac{t^{\alpha}}{\alpha \Gamma(\alpha)}=\frac{t^{\alpha}}{\Gamma(\alpha+1)}, t \geq 0
$$

If we use the inequalities (44) and 45 we get

$$
\begin{aligned}
& \quad \mid I_{g, a+, b-}^{\alpha} f(x) \\
& -\frac{1}{2 \Gamma(\alpha+1)}\left[\frac{f(x)+f(b)}{2}(g(b)-g(x))^{\alpha}+\frac{f(a)+f(x)}{2}(g(x)-g(a))^{\alpha}\right] \mid \\
& \leq \frac{1}{4 \Gamma(\alpha+1)}\left[(g(x)-g(a))^{\alpha} \bigvee_{a}^{x}(f)+(g(b)-g(x))^{\alpha} \bigvee_{x}^{b}(f)\right] \\
& \leq \frac{1}{4 \Gamma(\alpha+1)} \\
& \times\left\{\begin{array}{l}
{\left[\frac{g(b)-g(a)}{2}+\left|g(x)-\frac{g(b)+g(a)}{2}\right|\right]^{\alpha} \bigvee_{a}^{b}(f) ;} \\
{\left[(g(b)-g(x))^{p \alpha}+(g(x)-g(a))^{p \alpha}\right]^{1 / p}\left(\left(\bigvee_{a}^{x}(f)\right)^{q}+\left(\bigvee_{x}^{b}(f)\right)^{q}\right)^{1 / q}} \\
{\left[(g(b)-g(x))^{\alpha}+(g(x)-g(a))^{\alpha}\right]\left[\frac{1}{2} \bigvee_{a}^{b}(f)+\frac{1}{2}\left|\bigvee_{a}^{x}(f)-\bigvee_{x}^{b}(f)\right|\right]}
\end{array}\right.
\end{aligned}
$$

and

$$
\begin{aligned}
& \mid \breve{I}_{g, a+, b-}^{\alpha} f(x) \\
& -\frac{1}{2 \Gamma(\alpha+1)}\left[\frac{f(x)+f(b)}{2}(g(b)-g(x))^{\alpha}+\frac{f(a)+f(x)}{2}(g(x)-g(a))^{\alpha}\right] \mid \\
& \leq \frac{1}{4 \Gamma(\alpha+1)}\left[(g(x)-g(a))^{\alpha} \bigvee_{a}^{x}(f)+(g(b)-g(x))^{\alpha} \bigvee_{x}^{b}(f)\right] \\
& \leq \frac{1}{4 \Gamma(\alpha+1)}
\end{aligned}
$$




$$
\times\left\{\begin{array}{l}
{\left[\frac{g(b)-g(a)}{2}+\left|g(x)-\frac{g(b)+g(a)}{2}\right|\right]^{\alpha} \bigvee_{a}^{b}(f)} \\
{\left[(g(b)-g(x))^{p \alpha}+(g(x)-g(a))^{p \alpha}\right]^{1 / p}\left(\left(\bigvee_{a}^{x}(f)\right)^{q}+\left(\bigvee_{x}^{b}(f)\right)^{q}\right)^{1 / q}} \\
\text { with } p, q>1, \frac{1}{p}+\frac{1}{q}=1 ; \\
{\left[(g(b)-g(x))^{\alpha}+(g(x)-g(a))^{\alpha}\right]\left[\frac{1}{2} \bigvee_{a}^{b}(f)+\frac{1}{2}\left|\bigvee_{a}^{x}(f)-\bigvee_{x}^{b}(f)\right|\right]}
\end{array}\right.
$$

for $x \in(a, b)$.

From (48) and 49 we get

$$
\begin{array}{r}
\left|I_{g, a+, b-}^{\alpha} f\left(M_{g}(a, b)\right)-\frac{(g(b)-g(a))^{\alpha}}{2^{\alpha+1} \Gamma(\alpha+1)}\left[f\left(M_{g}(a, b)\right)+\frac{f(a)+f(b)}{2}\right]\right| \\
\leq \frac{1}{2^{\alpha+2} \Gamma(\alpha+1)}(g(b)-g(a))^{\alpha} \bigvee_{a}^{b}(f)
\end{array}
$$

and

$$
\begin{array}{r}
\left|\breve{I}_{g, a+, b-}^{\alpha} f\left(M_{g}(a, b)\right)-\frac{(g(b)-g(a))^{\alpha}}{2^{\alpha+1} \Gamma(\alpha+1)}\left[f\left(M_{g}(a, b)\right)+\frac{f(a)+f(b)}{2}\right]\right| \\
\leq \frac{1}{2^{\alpha+2} \Gamma(\alpha+1)}(g(b)-g(a))^{\alpha} \bigvee_{a}^{b}(f) .
\end{array}
$$

\section{Example for an Exponential Kernel}

For $\alpha, \beta \in \mathbb{R}$ we consider the kernel $k(t):=\exp [(\alpha+\beta i) t], t \in \mathbb{R}$. We have

$$
K(t)=\frac{\exp [(\alpha+\beta i) t]-1}{(\alpha+\beta i)}, \text { if } t \in \mathbb{R}
$$

for $\alpha, \beta \neq 0$.

Also, we have

$$
|k(s)|:=|\exp [(\alpha+\beta i) s]|=\exp (\alpha s) \text { for } s \in \mathbb{R}
$$

and

$$
\mathbf{K}(t)=\int_{0}^{t} \exp (\alpha s) d s=\frac{\exp (\alpha t)-1}{\alpha} \text { if } 0<t
$$

for $\alpha \neq 0$.

Let $f:[a, b] \rightarrow \mathbb{C}$ be a function of bounded variation on $[a, b]$ and $g$ be a strictly increasing function on $(a, b)$, having a continuous derivative $g^{\prime}$ on $(a, b)$. We consider the operator

$$
\mathcal{H}_{g, a+, b-}^{\alpha+\beta i} f(x):=\frac{1}{2} \int_{a}^{x} \exp [(\alpha+\beta i)(g(x)-g(t))] g^{\prime}(t) f(t) d t
$$




$$
+\frac{1}{2} \int_{x}^{b} \exp [(\alpha+\beta i)(g(t)-g(x))] g^{\prime}(t) f(t) d t
$$

for $x \in(a, b)$.

If $g=\ln h$ where $h:[a, b] \rightarrow(0, \infty)$ is a strictly increasing function on $(a, b)$, having a continuous derivative $h^{\prime}$ on $(a, b)$, then we can consider the following operator as well

$$
\begin{aligned}
& \kappa_{h, a+, b-}^{\alpha+\beta i} f(x) \\
& :=\mathcal{H}_{\ln h, a+, b-}^{\alpha+\beta i} f(x) \\
& =\frac{1}{2}\left[\int_{a}^{x}\left(\frac{h(x)}{h(t)}\right)^{\alpha+\beta i} \frac{h^{\prime}(t)}{h(t)} f(t) d t+\int_{x}^{b}\left(\frac{h(t)}{h(x)}\right)^{\alpha+\beta i} \frac{h^{\prime}(t)}{h(t)} f(t) d t\right]
\end{aligned}
$$

for $x \in(a, b)$.

Using the inequality 44 we have for $x \in(a, b)$

$$
\begin{aligned}
& \mid \mathcal{H}_{g, a+, b-}^{\alpha+\beta i} f(x)-\frac{1}{2} \frac{f(x)+f(b)}{2} \frac{\exp [(\alpha+\beta i)(g(b)-g(x))]-1}{(\alpha+\beta i)} \\
& -\frac{f(a)+f(x)}{2} \frac{\exp [(\alpha+\beta i)(g(x)-g(a))]-1}{(\alpha+\beta i)} \mid \\
& \leq \frac{1}{4}\left[\frac{\exp (\alpha(g(x)-g(a)))-1}{\alpha} \bigvee_{a}^{x}(f)+\frac{\exp (\alpha(g(b)-g(x)))-1}{\alpha} \bigvee_{x}^{b}(f)\right] \\
& \leq \frac{1}{4}\left\{\begin{array}{l}
\max \left\{\frac{\exp (\alpha(g(x)-g(a)))-1}{\alpha}, \frac{\exp (\alpha(g(b)-g(x))}{\alpha}\right. \\
{\left[\left(\frac{\exp (\alpha(g(x)-g(a)))-1}{\alpha}\right)^{p}+\left(\frac{\exp (\alpha(g(b)-g}{\alpha}\right.\right.} \\
\times\left(\left(\bigvee_{a}^{x}(f)\right)^{q}+\left(\bigvee_{x}^{b}(f)\right)^{q}\right)^{1 / q} \\
\text { with } p, q>1, \frac{1}{p}+\frac{1}{q}=1 ; \\
{\left[\frac{\exp (\alpha(g(x)-g(a)))+\exp (\alpha(g(b)-g(x)))-2}{\alpha}\right]} \\
\times\left[\frac{1}{2} \bigvee_{a}^{b}(f)+\frac{1}{2}\left|\bigvee_{a}^{x}(f)-\bigvee_{x}^{b}(f)\right|\right]
\end{array}\right.
\end{aligned}
$$

and if we take $g=\ln h$ where $h:[a, b] \rightarrow(0, \infty)$ is a strictly increasing function on $(a, b)$, having a continuous derivative $h^{\prime}$ on $(a, b)$, then we get

$$
\mid \kappa_{h, a+, b-}^{\alpha+\beta i} f(x)-\frac{1}{2}\left[\frac{f(x)+f(b)}{2} \frac{\left(\frac{h(b)}{h(x)}\right)^{\alpha+\beta i}-1}{(\alpha+\beta i)}\right.
$$




$$
\begin{gathered}
\left.-\frac{f(a)+f(x)}{2} \frac{\left(\frac{h(x)}{h(a)}\right)^{\alpha+\beta i}-1}{(\alpha+\beta i)}\right] \mid \\
\leq \frac{1}{4}\left[\frac{\left(\frac{h(x)}{h(a)}\right)^{\alpha}-1}{\alpha} \bigvee_{a}^{x}(f)+\frac{\left(\frac{h(b)}{h(x)}\right)^{\alpha}-1}{\alpha} \bigvee_{x}^{b}(f)\right] \\
\leq \frac{1}{4}\left\{\begin{array}{l}
\max \left\{\frac{\left(\frac{h(x)}{h(a)}\right)^{\alpha}-1}{\alpha}, \frac{\left(\frac{h(b)}{h(x)}\right)^{\alpha}-1}{\alpha}\right\} \bigvee_{a}^{b}(f) ; \\
{\left[\left(\frac{\left(\frac{h(x)}{h(a)}\right)^{\alpha}-1}{\alpha}\right)^{p}+\left(\frac{\left(\frac{h(b)}{h(x)}\right)^{\alpha}-1}{\alpha}\right)^{p}\right]^{1 / p}\left(\left(\bigvee_{a}^{x}(f)\right)^{q}+\left(\bigvee_{x}^{b}(f)\right)^{q}\right)^{1 / q}} \\
\operatorname{with} p, q>1, \frac{1}{p}+\frac{1}{q}=1 ; \\
{\left[\frac{\left(\frac{h(x)}{h(a)}\right)^{\alpha}+\left(\frac{h(b)}{h(x)}\right)^{\alpha}-2}{\alpha}\right]\left[\frac{1}{2} \bigvee_{a}^{b}(f)+\frac{1}{2}\left|\bigvee_{a}^{x}(f)-\bigvee_{x}^{b}(f)\right|\right] .}
\end{array}\right.
\end{gathered}
$$

If we take if we take $x_{h}:=h^{-1}(\sqrt{h(a) h(b)})=h^{-1}(G(h(a), h(b))) \in(a, b)$, where $G$ is the geometric mean, then from 65) we get

$$
\begin{array}{r}
\left|\bar{\kappa}_{h, a+, b-}^{\alpha+\beta i} f-\frac{\left(\frac{h(b)}{h(a)}\right)^{\frac{\alpha+\beta i}{2}}-1}{2(\alpha+\beta i)}\left[f\left(h^{-1}(G(h(a), h(b)))\right)+\frac{f(a)+f(b)}{2}\right]\right| \\
\leq \frac{1}{4} \frac{\left(\frac{h(b)}{h(a)}\right)^{\frac{\alpha}{2}}-1}{\alpha} \bigvee_{a}^{b}(f),
\end{array}
$$

where $\bar{\kappa}_{h, a+, b-}^{\alpha+\beta i} f=\kappa_{h, a+, b-}^{\alpha+\beta i} f\left(x_{h}\right)$.

Let $f:[a, b] \rightarrow \mathbb{C}$ be an integrable function on $[a, b]$ and $g$ be a strictly increasing function on $(a, b)$, having a continuous derivative $g^{\prime}$ on $(a, b)$. Also define

$$
\begin{aligned}
& \breve{\mathcal{H}}_{g, a+, b-}^{\alpha} f(x) \\
& :=\frac{1}{2} \int_{x}^{b} \exp [\alpha(g(b)-g(t))] g^{\prime}(t) f(t) d t \\
& +\frac{1}{2} \int_{a}^{x} \exp [\alpha(g(t)-g(a))] g^{\prime}(t) f(t) d t
\end{aligned}
$$

for any $x \in(a, b)$.

If $g=\ln h$ where $h:[a, b] \rightarrow(0, \infty)$ is a strictly increasing function on $(a, b)$, having a continuous derivative $h^{\prime}$ on $(a, b)$, then we can consider the following 
operator as well

$$
\begin{aligned}
& \breve{\kappa}_{h, a+, b-}^{\alpha} f(x) \\
& :=\breve{\mathcal{H}}_{\ln h, a+, b-}^{\alpha} f(x) \\
& =\frac{1}{2}\left[\int_{x}^{b}\left(\frac{h(b)}{h(t)}\right)^{\alpha} \frac{h^{\prime}(t)}{h(t)} f(t) d t+\int_{a}^{x}\left(\frac{h(t)}{h(a)}\right)^{\alpha} \frac{h^{\prime}(t)}{h(t)} f(t) d t\right],
\end{aligned}
$$

for any $x \in(a, b)$.

Using the inequality 45 we have for $x \in(a, b)$ that

$$
\begin{aligned}
& \mid \breve{\mathcal{H}}_{g, a+, b-}^{\alpha+\beta i} f(x)-\frac{1}{2}\left[\frac{f(x)+f(b)}{2} \frac{\exp [(\alpha+\beta i)(g(b)-g(x))]-1}{(\alpha+\beta i)}\right. \\
& \left.-\frac{f(a)+f(x)}{2} \frac{\exp [(\alpha+\beta i)(g(x)-g(a))]-1}{(\alpha+\beta i)}\right] \mid \\
& \leq \frac{1}{4}\left[\frac{\exp (\alpha(g(x)-g(a)))-1}{\alpha} \bigvee_{a}^{x}(f)+\frac{\exp (\alpha(g(b)-g(x)))-1}{\alpha} \bigvee_{x}^{b}(f)\right] \\
& \leq \frac{1}{4}\left\{\begin{array}{l}
\max \left\{\frac{\exp (\alpha(g(x)-g(a)))-1}{\alpha}, \frac{\exp (\alpha(g(b)-g(x)}{\alpha}\right. \\
{\left[\left(\frac{\exp (\alpha(g(x)-g(a)))-1}{\alpha}\right)^{p}+\left(\frac{\exp (\alpha(g(b)-}{\alpha}\right.\right.} \\
\times\left(\left(\bigvee_{a}^{x}(f)\right)^{q}+\left(\bigvee_{x}^{b}(f)\right)^{q}\right)^{1 / q} \\
\text { with } p, q>1, \frac{1}{p}+\frac{1}{q}=1 ; \\
{\left[\frac{\exp (\alpha(g(x)-g(a)))+\exp (\alpha(g(b)-g(x)))-2}{\alpha}\right]} \\
\times\left[\frac{1}{2} \bigvee_{a}^{b}(f)+\frac{1}{2}\left|\bigvee_{a}^{x}(f)-\bigvee_{x}^{b}(f)\right|\right]
\end{array}\right.
\end{aligned}
$$

and if we take $g=\ln h$ where $h:[a, b] \rightarrow(0, \infty)$ is a strictly increasing function on $(a, b)$, having a continuous derivative $h^{\prime}$ on $(a, b)$, then we get

$$
\begin{aligned}
& \breve{\kappa}_{h, a+, b-}^{\alpha+\beta i} f(x)-\frac{1}{2} {\left[\frac{f(x)+f(b)}{2} \frac{\left(\frac{h(b)}{h(x)}\right)^{\alpha+\beta i}-1}{(\alpha+\beta i)}\right.} \\
&\left.-\frac{f(a)+f(x)}{2} \frac{\left(\frac{h(x)}{h(a)}\right)^{\alpha+\beta i}-1}{(\alpha+\beta i)}\right] \mid \\
& \leq \frac{1}{4}\left[\frac{\left(\frac{h(x)}{h(a)}\right)^{\alpha}-1}{\alpha} \bigvee_{a}^{x}(f)+\frac{\left(\frac{h(b)}{h(x)}\right)^{\alpha}-1}{\alpha} \bigvee_{x}^{b}(f)\right]
\end{aligned}
$$




$$
\leq \frac{1}{4}\left\{\begin{array}{l}
\max \left\{\frac{\left(\frac{h(x)}{h(a)}\right)^{\alpha}-1}{\alpha}, \frac{\left(\frac{h(b)}{h(x)}\right)^{\alpha}-1}{\alpha}\right\} \bigvee_{a}^{b}(f) ; \\
{\left[\left(\frac{\left(\frac{h(x)}{h(a)}\right)^{\alpha}-1}{\alpha}\right)^{p}+\left(\frac{\left(\frac{h(b)}{h(x)}\right)^{\alpha}-1}{\alpha}\right)^{p}\right]^{1 / p}\left(\left(\bigvee_{a}^{x}(f)\right)^{q}+\left(\bigvee_{x}^{b}(f)\right)^{q}\right)^{1 / q}} \\
\text { with } p, q>1, \frac{1}{p}+\frac{1}{q}=1 ; \\
{\left[\frac{\left(\frac{h(x)}{h(a)}\right)^{\alpha}+\left(\frac{h(b)}{h(x)}\right)^{\alpha}-2}{\alpha}\right]\left[\frac{1}{2} \bigvee_{a}^{b}(f)+\frac{1}{2}\left|\bigvee_{a}^{x}(f)-\bigvee_{x}^{b}(f)\right|\right] .}
\end{array}\right.
$$

If we take if we take $x_{h}=h^{-1}(G(h(a), h(b))) \in(a, b)$, where $G$ is the geometric mean, then from 65 we get

$$
\begin{array}{r}
\left|\bar{\ell}_{h, a+, b-}^{\alpha+\beta i} f-\frac{\left(\frac{h(b)}{h(a)}\right)^{\frac{\alpha+\beta i}{2}}-1}{2(\alpha+\beta i)}\left[f\left(h^{-1}(G(h(a), h(b)))\right)+\frac{f(a)+f(b)}{2}\right]\right| \\
\leq \frac{1}{4} \frac{\left(\frac{h(b)}{h(a)}\right)^{\frac{\alpha}{2}}-1}{\alpha} \bigvee_{a}^{b}(f),
\end{array}
$$

where $\bar{\ell}_{h, a+, b-}^{\alpha+\beta i} f=\breve{\kappa}_{h, a+, b-}^{\alpha+\beta i} f\left(x_{h}\right)$.

\section{REFERENCES}

[1] Agarwal, R. P., Luo, M.-J. and Raina, R. K., On Ostrowski type inequalities, Fasc. Math. 56 (2016), 5-27.

[2] Aljinović, A. Aglić, Montgomery identity and Ostrowski type inequalities for RiemannLiouville fractional integral. J. Math. 2014, Art. ID 503195, 6 pp.

[3] Apostol, T. M., Mathematical Analysis, Second Edition, Addison-Wesley Publishing Company, 1975.

[4] Akdemir, A. O., Inequalities of Ostrowski's type for $m$ - and $(\alpha, m)$-logarithmically convex functions via Riemann-Liouville fractional integrals. J. Comput. Anal. Appl. 16 (2014), no. $2,375-383$

[5] Anastassiou, G. A., Fractional representation formulae under initial conditions and fractional Ostrowski type inequalities. Demonstr. Math. 48 (2015), no. 3, 357-378

[6] Anastassiou, G. A., The reduction method in fractional calculus and fractional Ostrowski type inequalities. Indian J. Math. 56 (2014), no. 3, 333-357.

[7] Budak, H., Sarikaya, M. Z. and Set, E., Generalized Ostrowski type inequalities for functions whose local fractional derivatives are generalized s-convex in the second sense. J. Appl. Math. Comput. Mech. 15 (2016), no. 4, 11-21.

[8] Cerone, P. and Dragomir, S. S., Midpoint-type rules from an inequalities point of view. Handbook of analytic-computational methods in applied mathematics, 135-200, Chapman \& Hall/CRC, Boca Raton, FL, 2000.

[9] Dragomir, S. S., The Ostrowski's integral inequality for Lipschitzian mappings and applications. Comput. Math. Appl. 38 (1999), no. 11-12, 33-37.

[10] Dragomir, S. S., The Ostrowski integral inequality for mappings of bounded variation. Bull. Austral. Math. Soc. 60 (1999), No. 3, 495-508. 
[11] Dragomir, S. S., On the midpoint quadrature formula for mappings with bounded variation and applications. Kragujevac J. Math. 22 (2000), 13-19.

[12] Dragomir, S. S., On the Ostrowski's integral inequality for mappings with bounded variation and applications, Math. Ineq. Appl. 4 (2001), No. 1, 59-66. Preprint: RGMIA Res. Rep. Coll. 2 (1999), Art. 7, [Online: http://rgmia.org/papers/v2n1/v2n1-7.pdf]

[13] Dragomir, S. S., Refinements of the generalised trapezoid and Ostrowski inequalities for functions of bounded variation. Arch. Math. (Basel) 91 (2008), no. 5, 450-460.

[14] Dragomir, S. S., Refinements of the Ostrowski inequality in terms of the cumulative variation and applications, Analysis (Berlin) 34 (2014), No. 2, 223-240. Preprint: RGMIA Res. Rep. Coll. 16 (2013), Art. 29 [Online:http://rgmia.org/papers/v16/v16a29.pdf] .

[15] Dragomir, S. S., Ostrowski type inequalities for Lebesgue integral: a survey of recent results, Australian J. Math. Anal. Appl., Volume 14, Issue 1, Article 1, pp. 1-287, 2017. [Online http://ajmaa.org/cgi-bin/paper.pl?string=v14n1/V14I1P1.tex] .

[16] Dragomir, S. S., Ostrowski type inequalities for Riemann-Liouville fractional integrals of bounded variation, Hölder and Lipschitzian functions, Preprint RGMIA Res. Rep. Coll. 20 (2017), Art. 48. [Online http://rgmia.org/papers/v20/v20a48.pdf] .

[17] Dragomir, S. S., Ostrowski type inequalities for generalized Riemann-Liouville fractional integrals of functions with bounded variation, RGMIA Res. Rep. Coll. 20 (2017), Art. 58. [Online http://rgmia.org/papers/v20/v20a58.pdf] .

[18] Dragomir, S. S., Further Ostrowski and trapezoid type inequalities for the generalized Riemann-Liouville fractional integrals of functions with bounded variation, RGMIA Res. Rep. Coll. 20 (2017), Art. 84. [Online http://rgmia.org/papers/v20/v20a84.pdf] .

[19] Dragomir, S. S., Ostrowski and trapezoid type inequalities for the generalized $k$ - $g$-fractional integrals of functions with bounded variation, RGMIA Res. Rep. Coll. 20 (2017), Art. 111. [Online http://rgmia.org/papers/v20/v20a111.pdf].

[20] Dragomir, S. S., Some inequalities for the generalized $k$ - $g$-fractional integrals of functions under complex boundedness conditions, RGMIA Res. Rep. Coll. 20 (2017), Art. 119. [Online http://rgmia.org/papers/v20/v20a119.pdf].

[21] Guezane-Lakoud, A. and Aissaoui, F., New fractional inequalities of Ostrowski type. Transylv. J. Math. Mech. 5 (2013), no. 2, 103-106

[22] Kashuri, A. and Liko, R., Ostrowski type fractional integral inequalities for generalized $(s, m, \varphi)$-preinvex functions. Aust. J. Math. Anal. Appl. 13 (2016), no. 1, Art. 16, 11 pp.

[23] Kilbas, A., Srivastava, H. M. and Trujillo, J. J., Theory and Applications of Fractional Differential Equations. North-Holland Mathematics Studies, 204. Elsevier Science B.V., Amsterdam, 2006. xvi+523 pp. ISBN: 978-0-444-51832-3; 0-444-51832-0.

[24] Kirane, M. and Torebek, B. T., Hermite-Hadamard, Hermite-Hadamard-Fejer, DragomirAgarwal and Pachpatte type Inequalities for convex functions via fractional integrals, Preprint arXiv:1701.00092.

[25] Noor, M. A., Noor, K. I. and Iftikhar, S., Fractional Ostrowski inequalities for harmonic h-preinvex functions. Facta Univ. Ser. Math. Inform. 31 (2016), no. 2, 417-445

[26] Raina, R. K., On generalized Wright's hypergeometric functions and fractional calculus operators, East Asian Math. J., 21(2)(2005), 191-203.

[27] Sarikaya, M. Z. and Filiz, H., Note on the Ostrowski type inequalities for fractional integrals. Vietnam J. Math. 42 (2014), no. 2, 187-190

[28] Sarikaya, M. Z. and Budak, H., Generalized Ostrowski type inequalities for local fractional integrals. Proc. Amer. Math. Soc. 145 (2017), no. 4, 1527-1538.

[29] Set, E., New inequalities of Ostrowski type for mappings whose derivatives are s-convex in the second sense via fractional integrals. Comput. Math. Appl. 63 (2012), no. 7, 1147-1154.

[30] Tunç, M., On new inequalities for $h$-convex functions via Riemann-Liouville fractional integration, Filomat 27:4 (2013), 559-565. 
[31] Tunç, M., Ostrowski type inequalities for $m$ - and $(\alpha, m)$-geometrically convex functions via Riemann-Louville fractional integrals. Afr. Mat. 27 (2016), no. 5-6, 841-850.

[32] Yildirim, H. and Kirtay, Z., Ostrowski inequality for generalized fractional integral and related inequalities, Malaya J. Mat., 2(3)(2014), 322-329.

[33] Yildiz, C., Özdemir, E and Muhamet, Z. S., New generalizations of Ostrowski-like type inequalities for fractional integrals. Kyungpook Math. J. 56 (2016), no. 1, 161-172.

[34] Yue, H., Ostrowski inequality for fractional integrals and related fractional inequalities. Transylv. J. Math. Mech. 5 (2013), no. 1, 85-89.

Current address: Silvestru Sever Dragomir: Mathematics, College of Engineering \& Science Victoria University, PO Box 14428 Melbourne City, MC 8001, Australia.

DST-NRF Centre of Excellence in the Mathematical and Statistical Sciences, School of Computer Science \& Applied Mathematics, University of the Witwatersrand, Private Bag 3, Johannesburg 2050, South Africa

E-mail address: sever.dragomir@vu.edu.au

ORCID Address: https://orcid.org/0000-0003-2902-6805 